OPEN ACCESS

Edited by:

Eung-Soo Kim

Inha University, South Korea

Reviewed by:

Gabriela Olmedo-Alvarez,

Cinvestav Unidad Irapuato, Mexico

Vania Aparecida Vicente,

Universidade Federal do Paraná,

Brazil

*Correspondence:

Ming Sun

m98sun@mail.hzau.edu.cn

Specialty section:

This article was submitted to

Microbial Physiology and Metabolism,

a section of the journal

Frontiers in Microbiology

Received: 24 May 2018 Accepted: 03 September 2018

Published: 11 October 2018

Citation:

Yang W, Ruan L, Tao J, Peng D,

Zheng $J$ and Sun $M$ (2018) Single

Amino Acid Substitution

in Homogentisate Dioxygenase Affects Melanin Production in Bacillus

thuringiensis.

Front. Microbiol. 9:2242.

doi: 10.3389/fmicb.2018.02242

\section{Single Amino Acid Substitution in Homogentisate Dioxygenase Affects Melanin Production in Bacillus thuringiensis}

\author{
Wenjun Yang ${ }^{1}$, Lifang Ruan ${ }^{1}$, Jiangming Tao ${ }^{1}$, Donghai Peng ${ }^{1}$, Jinshui Zheng ${ }^{1,2}$ and \\ Ming Sun ${ }^{1 *}$ \\ 'State Key Laboratory of Agricultural Microbiology, College of Life Science and Technology, Huazhong Agricultural \\ University, Wuhan, China, ${ }^{2}$ College of Informatics, Huazhong Agricultural University, Wuhan, China
}

Bacillus thuringiensis formulation losing its activity under field conditions due to UV radiation and photoprotection of $B$. thuringiensis based on melanin has attracted the attention of researchers for many years. Here, a single amino acid substitution (G272E) in homogentisate 1,2-dioxygenase was found to be responsible for pigment overproduction in B. thuringiensis BMB181, a derivative of BMB171. Disrupting the gene encoding homogentisate dioxygenase in BMB171 induced the accumulation of the homogentisic acid and provoked an increased pigment formation. To gain insights into homogentisate 1,2-dioxygenase in B. thuringiensis, we constructed a total of 14 mutations with a single amino acid substitution, and six of the mutant proteins were found to affect the melanin production when substituted by alanine. This study provides a new way to construct pigment-overproducing strains by impairing the homogentisate dioxygenase with a single mutation in B. thuringiensis, and the findings will facilitate a better understanding of this enzyme.

Keywords: Bacillus thuringiensis, tyrosine catabolism, homogentisate dioxygenase, site-directed mutagenesis, melanin

\section{INTRODUCTION}

Bacillus thuringiensis, a gram-positive spore-forming soil bacterium, has been widely used in biological pest control due to the formation of parasporal crystal proteins that are toxic to the larvae of various insect pests (Sudakin, 2003). However, the insecticidal activity of the crystal proteins would be reduced or destroyed under field conditions because of UV damage in sunlight (Pusztai et al., 1991). To solve this problem, researchers have proposed a series of strategies to protect insecticidal crystal proteins from UV damage (Sanchis et al., 1999; Manasherob et al., 2002; Jallouli et al., 2014), and one of them is the use of melanin, a photoprotective agent, to reduce the damaging effect of UV radiation on B. thuringiensis toxins (Liu et al., 1993; Ruan et al., 2002; Zhang et al., 2008; Sansinenea and Ortiz, 2015). 
Melanins are polymers of phenolic and/or indolic compounds and classified into three main categories: eumelanins, pheomelanins, and allomelanins (Plonka and Grabacka, 2006). These black pigments are widely distributed in nature and can be found in species of all biological kingdoms, including humans, fungi, and bacteria (Plonka and Grabacka, 2006). Melanins provide free-living species a survival advantage in the environment by protecting against different exogenous stresses, such as UV-irradiation, reactive oxygen species (ROS), metals, and defensins (Nosanchuk and Casadevall, 2003, 2006; Heinekamp et al., 2012). Both eumelanins and pheomelanins are produced from the oxidation of tyrosine or phenylalanine to o-dihydroxyphenylalanine (DOPA) and dopaquinone via tyrosinases or laccases. Allomelanins include a heterogeneous group of polymers formed through the oxidation and polymerization of the intermediates such as dihydroxynaphthalene, homogentisic acid (HGA), $\gamma$-glutaminyl4-hydroxybenzene, catechols, and 4-hydroxyphenylacetic acid (Plonka and Grabacka, 2006).

Homogentisic acid is derived from the tyrosine or phenylalanine catabolism pathway (one branch of tyrosine metabolism) and further oxidized to acetoacetic acid and fumaric acid (Turick et al., 2010). Pyomelanin is formed from the autoxidation and self-polymerization of HGA with the deactivation of homogentisate dioxygenase (HmgA) or the disruption of the gene encoding HGA-oxidase (Rodriguez-Rojas et al., 2009; Schmaler-Ripcke et al., 2009; Valeru et al., 2009). A deficiency of this enzyme in humans causes the metabolic disease alkaptonuria (AKU), leading to the excretion of HGA in the urine in a large amount and its deposition in different tissues (Millucci et al., 2012). The synthesis of pyomelanin has been investigated in a broad range of bacteria, such as Aeromonas media, Burkholderia cepacia, Bacillus anthracis, Legionella pneumophila, Pseudoalteromonas, Pseudomonas aeruginosa, Pseudomonas putida, Ralstonia solanacearum, Shewanella colwelliana, and so on (Arias-Barrau et al., 2004; Valeru et al., 2009; Turick et al., 2010; Gonyar et al., 2015; Han et al., 2015; Wang et al., 2015; Ahmad et al., 2017; Zeng et al., 2017).

A number of studies have been performed to improve the photoprotection of the $B$. thuringiensis crystal proteins by producing melanin or increasing the melanin yield in B. thuringiensis cells. Some studies focused on screening B. thuringiensis mutants that can produce melanin in different conditions, and others attempted to construct recombinant B. thuringiensis strains with melanin production by genetic engineering (Hoti and Balaraman, 1993; Ruan et al., 2002; Saxena et al., 2002; Zhang et al., 2008; Sansinenea and Ortiz, 2015). It has been found that melanin could be produced by most B. thuringiensis strains in the presence of L-tyrosine at an elevated temperature $\left(42^{\circ} \mathrm{C}\right)$, but the insecticidal Cry proteins could not be synthesized at this temperature (Ruan et al., 2004). In our previous work, a mutant, $B$. thuringiensis strain BMB181, was identified to be able to produce the brownish black pigment as an alternative melanin without tyrosine supplementation in the growth medium, and this strain could achieve a high melanin yield in different media without additional L-tyrosine (Liu et al., 2013). However, the mechanism for melanin production by BMB181 remains unclear. Here, the pigment produced by the strain BMB181 was found to be derived from homogentisate acid. The inactivation of HmgA by a G272E amino acid substitution resulted in pigmentation in the strain BMB181. Six single-point mutations in HmgA resulted in observable changes of melanin production in B. thuringiensis. This study offers valuable information about HmgA and provides a new way of constructing the pigment production strain of $B$. thuringiensis.

\section{MATERIALS AND METHODS}

\section{Bacterial Strains, Plasmids, and Growth Conditions}

The bacterial strains and plasmids used in this study are shown in Table 1. The B. thuringiensis strain BMB171 and its derivative BMB181 have been reported in previous studies (He et al., 2010; Liu et al., 2013). Bacteria were grown in Luria-Bertani (LB) medium at $37^{\circ} \mathrm{C}$ (E. coli) or $28^{\circ} \mathrm{C}$ (B. thuringiensis) and $200 \mathrm{rpm}$. The antibiotics, including kanamycin $(50 \mu \mathrm{g} / \mathrm{mL})$, erythromycin (25 $\mu \mathrm{g} / \mathrm{mL})$, and ampicillin $(100 \mu \mathrm{g} / \mathrm{mL})$, were added when necessary.

\section{DNA Manipulation and Sequence Analysis}

In this study, DNA was manipulated using the standard techniques as previously described (Green and Sambrook, 2012). The DNA of $B$. thuringiensis was extracted as previously reported (Andrup et al., 1993). The DNA fragments were amplified with the related primers, and the polymerase chain reaction (PCR) products were confirmed by DNA sequencing. The DNA sequences were analyzed using the DNASTAR software and the protein sequences were compared with those of other proteins using BLAST and CLUSTALX.

\section{Transformation Techniques}

Escherichia coli was transformed using $\mathrm{CaCl}_{2}$-treated competent cells (Green and Sambrook, 2012), and B. thuringiensis was transformed by electroporation with the Bio-Rad Gene Pulser set (Bio-Rad, Hercules, CA, United States) (Peng et al., 2009).

\section{High Performance Liquid Chromatography (HPLC) Analysis of Culture Filtrates}

The HPLC analysis of culture filtrates was performed using an HPLC apparatus equipped with a variable wavelength UVvisible detector (CapLC 2487, Waters) and a C-18 end-capped column $(10 \mu \mathrm{m}, 4.6 \mathrm{~mm} \times 150 \mathrm{~mm}$; Elite). Briefly, the bacteria were grown in $\mathrm{LB}$ medium at $28^{\circ} \mathrm{C}$ and $200 \mathrm{rpm}$, and the culture supernatants were collected by centrifugation and filter sterilization. Next, $20 \mu \mathrm{L}$ of the sample was injected into the column for HPLC. The mobile phase was $50 \mathrm{mM}$ sodium phosphate buffer $(\mathrm{pH} 6.5) /$ methanol $(80: 20, \mathrm{v} / \mathrm{v})$ at a flow rate of $0.5 \mathrm{~mL} / \mathrm{min}$ as described by Fernandez-Canon and Penalva (1995). The chromatograms of standard solutions of HGA (from Sigma) were used as a reference to identify the corresponding HPLC peaks. The absorption maximum of HGA is $290 \mathrm{~nm}$. 
TABLE 1 | Bacterial strains and plasmids used in this study.

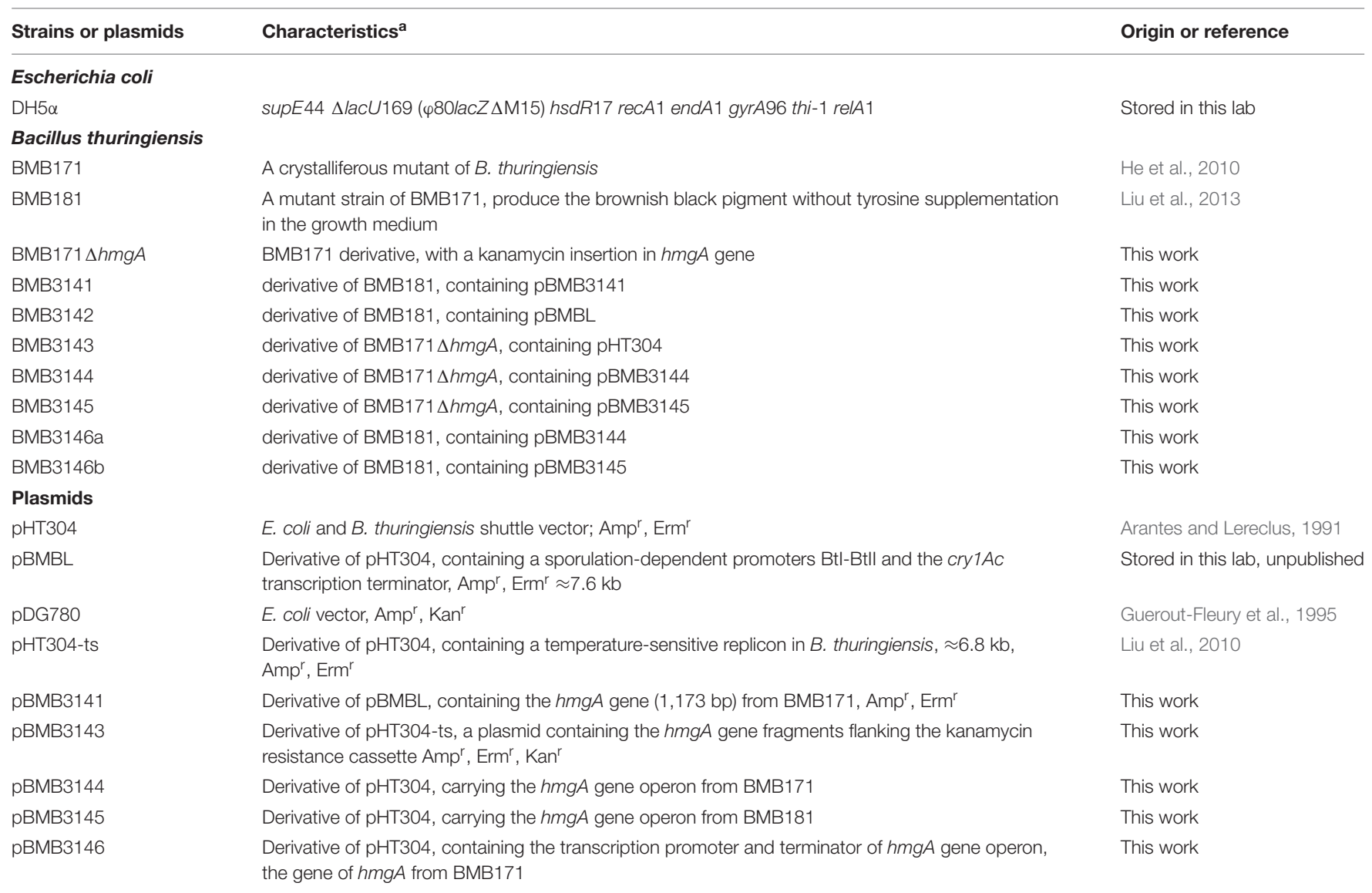

${ }^{a} \mathrm{Amp}^{r}$, ampicillin resistance; Erm ${ }^{r}$, erythromycin resistance; Kan', kanamycin resistance.

\section{Isolation of the $h m g A$ Gene}

The $h m g A$ gene ( $1,173 \mathrm{bp}$ ) encoding HmgA was amplified from B. thuringiensis BMB171 and BMB181 genomic DNA using the pair of primers, hmgAl (5'-CGCGGATCCATGTTTTATCGT CACATGGGAG-3', with the BamHI recognition sequence underlined) and hmgA2 (5'-CGCGTCGACTTATTTCACAG TATATGAACCT-3', with the SalI recognition sequence underlined). The $h m g A$ genes from BMB171 and BMB181 were designated as $171 \mathrm{hmgA}$ and $181 \mathrm{hmgA}$, respectively.

\section{Insertional Inactivation of the $h m g A$ Gene}

To verify the function of the $h m g A$ gene, the gene disruption strain was constructed for BMB171 via homologous recombination using a temperature-sensitive shuttle vector pHT304-ts containing the temperature-sensitive replication origin (Liu et al., 2010). Briefly, a 503-bp fragment and a 600-bp fragment, corresponding to the DNA regions upstream and downstream of the open reading frame of the $h m g A$ gene in the strain BMB171, were generated by PCR using the primer pairs HmgA-up-1 (5'-CGCGGATCCTGGGAGAACTACCTCATAAAC-3')/HmgA -up-2(5'-CCGGAATTCGCTATTCGCCTCTACAACA-3') and HmgA-down-1 (5'-CCGGTCGACAATTGTTAGAGCATAGTCC G-3')/HmgA-down-2(5'-CGGGGTACCATGAACCTTGTTCAA
TCCAG-3') and digested with BamHI- EcoRI and AccI-KpnI, respectively. A kanamycin resistance cassette (1,514 bp) was acquired by digesting plasmid pDG780 (Guerout-Fleury et al., 1995) with EcoRI-AccI. These three fragments were cloned into the plasmid pHT304-ts at the BamHI-KpnI site. The resulting plasmid, pBMB3143, was transformed into the strain BMB171 by electroporation.

The mutants were selected as follows. Specifically, the transformants were cultured in LB medium with kanamycin $(50 \mu \mathrm{g} / \mathrm{mL})$ for $8 \mathrm{~h}$, followed by incubation at $42^{\circ} \mathrm{C}$ for 4 days to eliminate unintegrated temperature-sensitive plasmids. Finally, the mutant strains that were resistant to kanamycin but sensitive to erythromycin colonies were confirmed by PCR using appropriate primers and sequencing.

\section{Genetic Complementation Analysis}

For genetic complementation analysis, complementation plasmids were prepared by using the shuttle vector pHT304 (Arantes and Lereclus, 1991) and its derivative pBMBL (unpublished data) that contained a sporulationdependent promoter BtI-BtII and the crylAc transcription terminator. The amplified fragment $171 \mathrm{hmgA}$ was cloned into the vector pBMBL to yield the plasmid pBMB3141. To construct the plasmids pBMB3144 (carrying 171hmgA 
operon) and pBMB3145 (carrying 181hmgA operon), the $h m g A$ gene operon region (about $3.9 \mathrm{~kb}$ ) containing the genes encoding 4-hydroxyphenylpyruvate dioxygenase (HppD), HmgA, and fumarylacetoacetate hydrolase (FahA) was amplified, respectively, from the strains BMB171 and BMB181 using special primers HMGAop-S (5' CGCGGATCCAGATATATAAATACAATCATTC-3', with the BamHI recognition sequence underlined) and HMGAop-A (5'-CGCGTCGACTCTTTCACTCCTCCAAGTTT-3', with the SalI recognition sequence underlined) and subsequently cloned into pHT304. Finally, the two recombinant plasmids were transformed into the pigmented $B$. thuringiensis strains by electroporation separately.

\section{Measurement of the Bacterial Growth Curve and the Pyomelanin Production}

The growth curve and the pigment production of the $B$. thuringiensis strains and their derivatives were evaluated according to optical density (OD). To monitor the growth curves of the strains, the bacteria were inoculated to $100 \mathrm{~mL}$ of $\mathrm{LB}$ medium (the flask volume is $500 \mathrm{~mL}$ ) and incubated under shaking at $28^{\circ} \mathrm{C}$ and $200 \mathrm{rpm}$, followed by the OD measurement of the cultures at $600 \mathrm{~nm}\left(\mathrm{OD}_{600}\right)$ at different time intervals. The melanin production of the strains was quantified by testing the absorbance of the centrifuged culture supernatant at $400 \mathrm{~nm}$ $\left(\mathrm{OD}_{400}\right)$ at the indicated time points (Ruan et al., 2002; Liu et al., 2013).

\section{Alanine Scanning Site-Directed Mutagenesis}

For performing alanine scanning site-directed mutagenesis, we constructed the plasmid pBMB3146 (a derivative of pHT304, containing the promoter region and the terminator region of the $h m g A$ gene operon and the $171 h m g A$ gene). Briefly, the promoter region of the $h m g A$ gene operon (394 bp) was amplified from the strain BMB171 genomic DNA using the pair of primers, HMGAop-S (5'-CGCGGATCCAGATATATAAATACA ATCATTC-3', with the BamHI recognition sequence under lined) and HMGAop-A2 (5'-CTCCCATGTGACGATAAAAC ATAATATCTTCATCTCCCTGTAA-3'). Next, the 1,405-bp PCR product, containing the $171 \mathrm{hmgA}$ gene and the terminator region of the $h m g A$ gene operon, was amplified using primers HMGA-S2 (5'-TTACAGGGAGATGAAGATATTATGTTTTAT CGTCACATGGGAG-3') and HMGAop-A (5'-CGCGTCGAC TCTTTCACTCCTCCAAGTTT-3', with the SalI recognition sequence underlined). Finally, the vector pBMB3146 was generated by connecting the two fragments with overlapping PCR (Higuchi et al., 1988) using primers HMGAop-S and HMGAop-A, digesting the overlapping PCR fragment with BamHI and SalI, and inserting it into the shuttle vector pHT304.

A total of fourteen amino acid residues of HmgA were mutated to alanine residues by overlapping PCR using the primers shown in Table 2, with vector pBMB3146 as the template. The PCR fragments were cloned separately into the vector pHT304 between the BamHI and SalI restriction sites. The recombinant plasmids were transformed into E. coli DH5a and the positive clones were screened by restriction enzyme analysis.
TABLE 2 | Primers used for site-directed mutagenesis.

\begin{tabular}{ll}
\hline Primers $\quad$ Oligonucleotides $\left(5^{\prime} \rightarrow 3^{\prime}\right)^{a}$ & Use
\end{tabular}

HMGAop-S CGCGGATCCAGATATATAAATACAATCATTC

HMGAOP-A CGCGTCGACTCTITCACTCCTCCAAGTIT

Hm89-R GTGATGCAATAAGTGCACGGAATITCATACT

G89A

substitution

Hm89-L AGTATGAAATTCCGTGCACTTATTGCATCAC

Hm116-R ATTATITCTATCGTGCAGGTGATGGCGACGA

N116A

substitution

Hm116-L TCGTCGCCATCACCTGCACGATAGAAATAAT

Hm119-R ATCGTAATGGTGATGCAGACGAAATGTTATT

G119A

substitution

Hm119-L AATAACATITCGTCTGCATCACCATTACGAT

Hm120-R GTAATGGTGATGGCGCAGAAATGTTATTTGT

D120A

substitution

Hm120-L ACAAATAACATTTCTGCGCCATCACCATTAC

Hm128-R TATTTGTCATTATGCAACAGGGAAATTGA

G128A

substitution

Hm128-L TCAATITTCCCTGTTGCATAATGAACAAATA

Hm136-R AAATTGAAACGATGGCAGGAACGATTCACTA

F136A

substitution

Hm136-L TAGTGAATCGTTCCTGCCATCGTITCAATIT

Hm219-R TTGTCGTAATGACAGCATCAAGAGGCTATAT

K219A

substitution

Hm219-L ATATAGCCTCTTGATGCTGTCATTACGACAA

Hm241-R TTGTGGGATGGGATGCATATTATATCCGTG

G241A

substitution

Hm241-L CACGGATATAAATATGCATCCCATCCCACAA

Hm245-R ATGGCTATITATATGCATGGGTATTTAATGT

P245A

substitution

Hm245-L ACATTAAATACCCATGCATATAAATAGCCAT

Hm261-R TTACAGGGCGCATTGCACAGCCACCGCCAGT

H261A

substitution

Hm261-L ACTGGCGGTGGCTGTGCAATGCGCCCTGTAA

Hm300-R CATATTATCATAGTGCAGTTAATAGTGATGA

N300A

substitution

Hm300-L TCATCACTATTAACTGCACTATGATAATATG

Hm323-R AAGGTGTGGAAGAAGCATCTATTACACTTCA

G323A

substitution

Hm323-L TGAAGTGTAATAGATGCTTCTTCCACACCTT

Hm334-R CGAGCGGGATTCCCGCAGGACCGCATCCGGG

H334A

substitution

Hm334-L CCCGGATGCGGTCCTGCGGGAATCCCGCTCG

Hm336-R GGATTCCCCATGGAGCACATCCGGGGAAAAC

P336A

substitution

Hm336-L GTITCCCCGGATGTGCTCCATGGGGAATCC

aThe restriction sites included in the oligonucleotide sequences are underlined. Nucleotide codons encoding the mutated amino acids are highlighted in bold types.

Before transformation into the pigmented $B$. thuringiensis strain BMB171 $\Delta h m g A$, all the resulting mutant plasmids were sequenced to ensure that the proper mutations were maintained.

\section{Sequence Accession Number}

The sequences reported in this paper have been submitted to the GenBank. The accession number for the complete genome of the strain BMB171 is CP001903.1. The accession number for the HmgA (locus tag is BMB171_C0216) is ADH05034.1. 

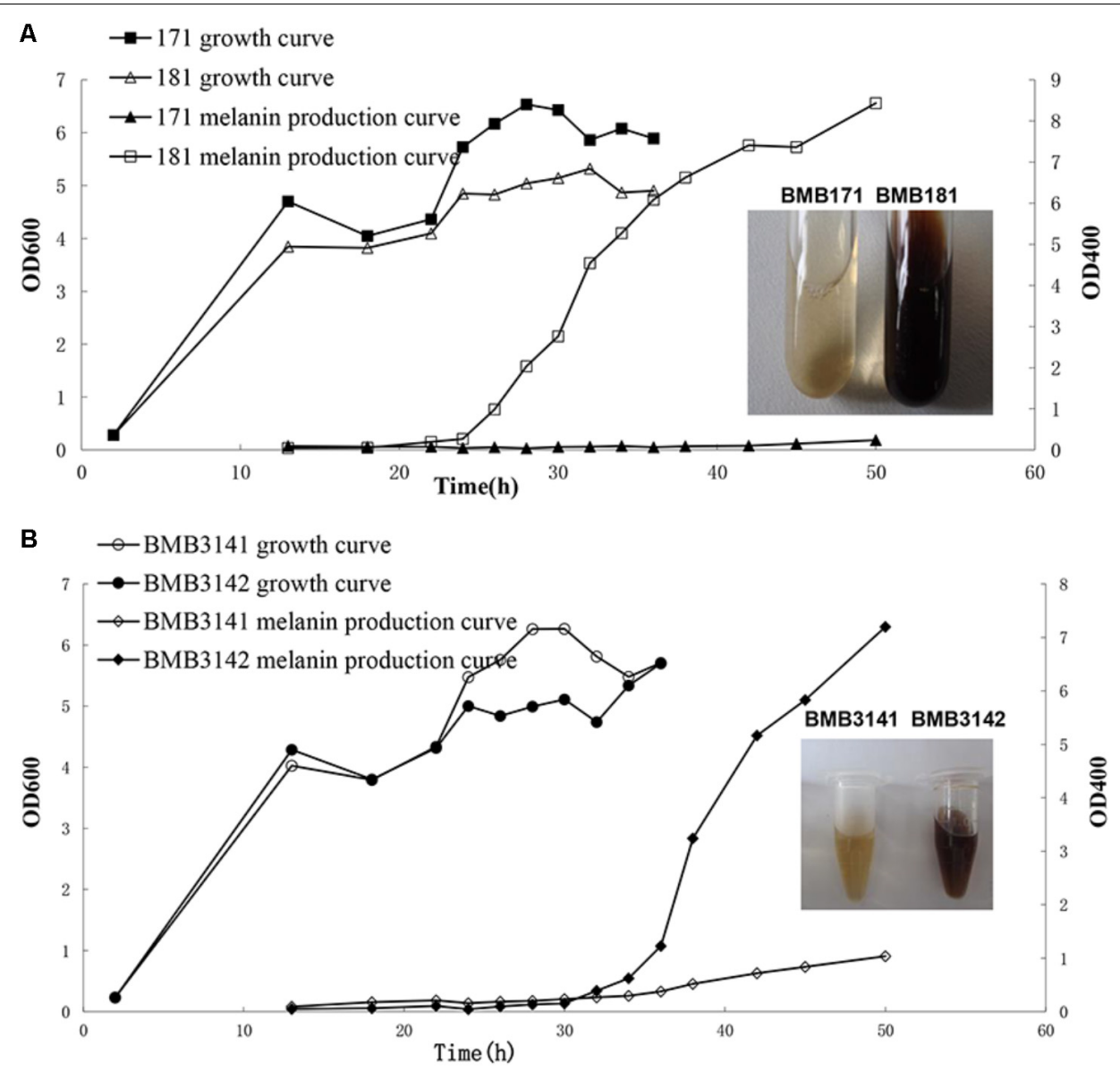

FIGURE 1 | Growth and melanin production curves of B. thuringiensis strains in LB medium. (A) Growth and melanin production curves of BMB171 and BMB181 in LB medium. (B) Growth and melanin production curves of BMB3141 and BMB3142 in LB medium. BMB3141, a derivative of BMB181, contained pBMB3141 that harbors 171 hmgA; BMB3142, a derivative of BMB181, contained pBMBL, as a negative control.

\section{RESULTS}

\section{Pigment Results From Polymerization of Homogentisate}

BMB181, a B. thuringiensis mutant with high melanin production, was obtained after subculturing the strain BMB171 for several generations at $42^{\circ} \mathrm{C}$ (Liu et al., 2013). The red pigment produced by the strain BMB181 turned dark brown with the extension of incubation time (Figure 1A). Pigments can be formed from the oxidation and polymerization of compounds such as DOPA and HGA (Plonka and Grabacka, 2006). In our early work, we found that the pigment produced by the strain BMB181 has nothing to do with DOPA (data not shown). Therefore, we test whether HGA is the precursor of pigment produced by the strain BMB181. Here, ascorbic acid was added as an antioxidant to prevent HGA from oxidation. No pigment was observed when ascorbic acid $(2 \mathrm{mM})$ was added to the cultures of the strain BMB181 (data not shown). Strains BMB171 and $\mathrm{BMB} 181$ were cultured in LB liquid media under shaking at $28^{\circ} \mathrm{C}$ for $24 \mathrm{~h}$ and the culture samples were taken for HPLC analysis after centrifugation and filtration, using the commercially available HGA as the standard. A peak corresponding to HGA (with a retention time of $8.68 \mathrm{~min}$ ) was identified in the culture supernatants of the strain BMB171 with HGA added to the culture during the logarithmic growth phase (Figures 2A,B). The peak with the same chromatography retention time as HGA was identified in the culture supernatants of the strain BMB181 (Figure 2C), suggesting that homogentisate could be secreted by the pigmented strain BMB181 and the pigment produced by the strain probably resulted from the accumulation and polymerization of homogentisate.

\section{Identification of Amino Acid Substitution in HmgA}

Considering that the melanin production from homogentisate induced by a deficiency of HmgA in organisms is associated with the tyrosine metabolism pathway (Figure 3A), we analyzed the genome of $\mathrm{BMB} 171$ and found that $\mathrm{BMB} 171$ carried the genes encoding HppD, HmgA, and FahA (Figure 3B). The relationship between the biosynthesis of the pigment in the strain BMB181 and HmgA was tested by amplifying the $h m g A$ gene from the BMB181 genomic DNA and sequencing. After aligning 
the inferred amino acid sequence of HmgA from the publicly available BMB171 sequences (He et al., 2010), a glycine was found to be replaced by a glutamate at residue 272 in $\mathrm{HmgA}$ in the pigmented strain BMB181.

Whether this amino acid substitution is responsible for the observed pigmented phenotype was tested by performing a complementation analysis. Specifically, the plasmid pBMB3141 containing the $171 \mathrm{hmgA}$ gene was transformed into BMB181 to create the transformant strain BMB3141 $\left(171 \mathrm{hmgA}^{+}\right)$, and $\mathrm{BMB} 3142$, a BMB181 derivative containing the plasmid $\mathrm{pBMBL}$ was used as a control. It was found that BMB3141 $\left(171 \mathrm{hmgA}^{+}\right)$ was reverted to a non-pigmented phenotype, with no significant increase observed in the $\mathrm{OD}_{400}$ of the supernatant relative to the control (Figure 1B). These results indicate that the G272E version of HmgA is responsible for melanin production in the B. thuringiensis pigmented strain BMB181.

\section{Disruption of HmgA Results in Pigment Formation Due to Inactivation of Homogentisate Dioxygenase}

To verify that the lack of a functional HmgA is responsible for the pigment formation of $B$. thuringiensis, a $h m g A$ insertion mutant strain was constructed by homologous recombination. Specifically, the plasmid pBMB3143 containing $h m g A$ gene fragments flanking the kanamycin resistance cassette was constructed and transferred into the strain BMB171. The recombinants with double-crossover homologous recombination integration in the resident $h m g A$ gene were selected and verified by PCR. The strain carrying the $h m g A:: k a n$ disruption, named BMB171 $h m g A$, was able to produce the brownish black pigment in cultures (Figure 4A). HPLC analysis showed that HGA was secreted and accumulated in the culture supernatants of BMB171 $\Delta h m g A$ (Figure 2D).

The complete $h m g A$ gene operons from the strains BMB171 and BMB181 were amplified and used for the complementation analysis, and the plasmids containing different alleles of $h m g A$ were named as pBMB3144 (carrying the operon containing the $h m g A$ gene from BMB171) and pBMB3145 (carrying the operon containing the $h m g A$ gene from BMB181). The plasmids expressing either allele of $h m g A$ were transferred into the strain BMB171 $\triangle h m g A$ (pigmented phenotype). It was found that the transformant harboring the $171 \mathrm{hmgA}$ gene operon (strain BMB3144) exhibited a non-pigmented phenotype, while the transformant harboring the $181 h m g A$ variant operon (strain BMB3145) remained pigmented (Figure 4B). The same result was found when the two plasmids were introduced into the strain BMB181 (Figure 4C). These data suggest that the HmgA variant from the BMB181 mutant with residue Gly272 replaced by the residue Glu is not functional and ultimately results in pigment overproduction, while the functional HmgA enzyme from the strain BMB171 results in the absence of pigment from HGA.

\section{Mutational Analysis of HmgA in B. thuringiensis}

To further test whether other single residue mutations have the same effect as G272E on melanin production, we designed 14

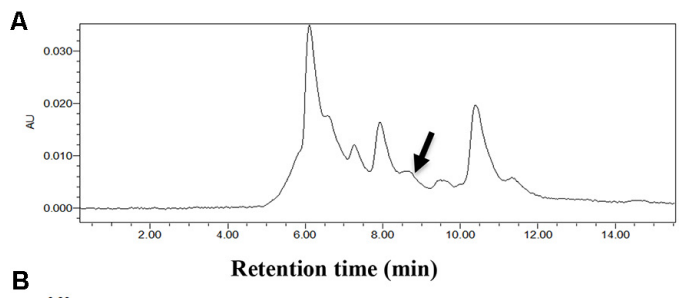

B
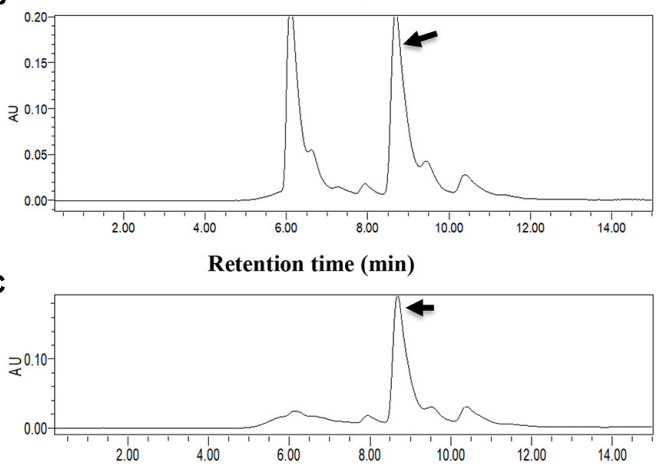

D

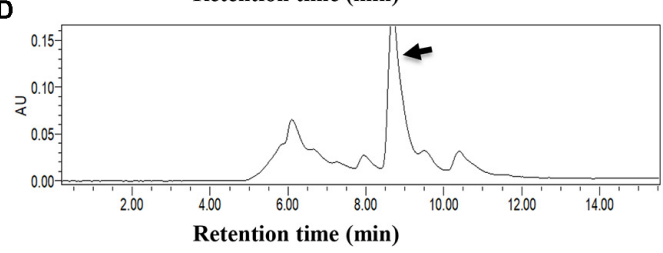

FIGURE 2 | HPLC analysis of culture filtrates of BMB171, BMB181, and $\mathrm{BMB} 171 \Delta h m g A$. Homogentisate acid was identified by HPLC and is indicated by black arrows. (A) Supernatants of BMB171 (with ascorbic acid), as a negative control. (B) Supernatants of BMB171 (with ascorbic acid and Homogentisate), as positive control. (C) Supernatants of BMB181 (with ascorbic acid). (D) Supernatants of BMB171 $\mathrm{hmgA}$ (with ascorbic acid).

single-point mutations (G89A, N116A, G119A, D120A, G128A, F136A, K219A, G241A, P245A, H261A, N300A, G323A, H334A, and $\mathrm{P} 336 \mathrm{~A}$ ) in $171 \mathrm{HmgA}$ based on the sequence alignment and the secondary structure of $171 \mathrm{HmgA}$ (Figure 5). A transcomplementation test was performed by introducing all the sequences under the control of the shuttle vector pHT304 separately into the pigmented strain BMB171 $\triangle \mathrm{hmgA}$. Among the fourteen transformants, eight (containing G89A, N116A, G119A, D120A, K219A, P245A, N300A, and G323A, respectively) showed a non-pigmented phenotype, while the other six (containing G128A, F136A, G241A, H261A, H334A, and P336A, respectively) showed a pigmented phenotype (Table 3 ), implying that these six amino acid substitutions could result in the function loss of HmgA, making it unable to restore the non-pigment phenotype.

\section{DISCUSSION}

In aerobic organisms, L-Tyrosine degradation via homogentisate (HGA) is initiated by the conversion of tyrosine to 4hydroxyphenylpyruvate by tyrosine aminotransferases, followed by the formation of HGA from 4-hydroxyphenylpyruvate 
A

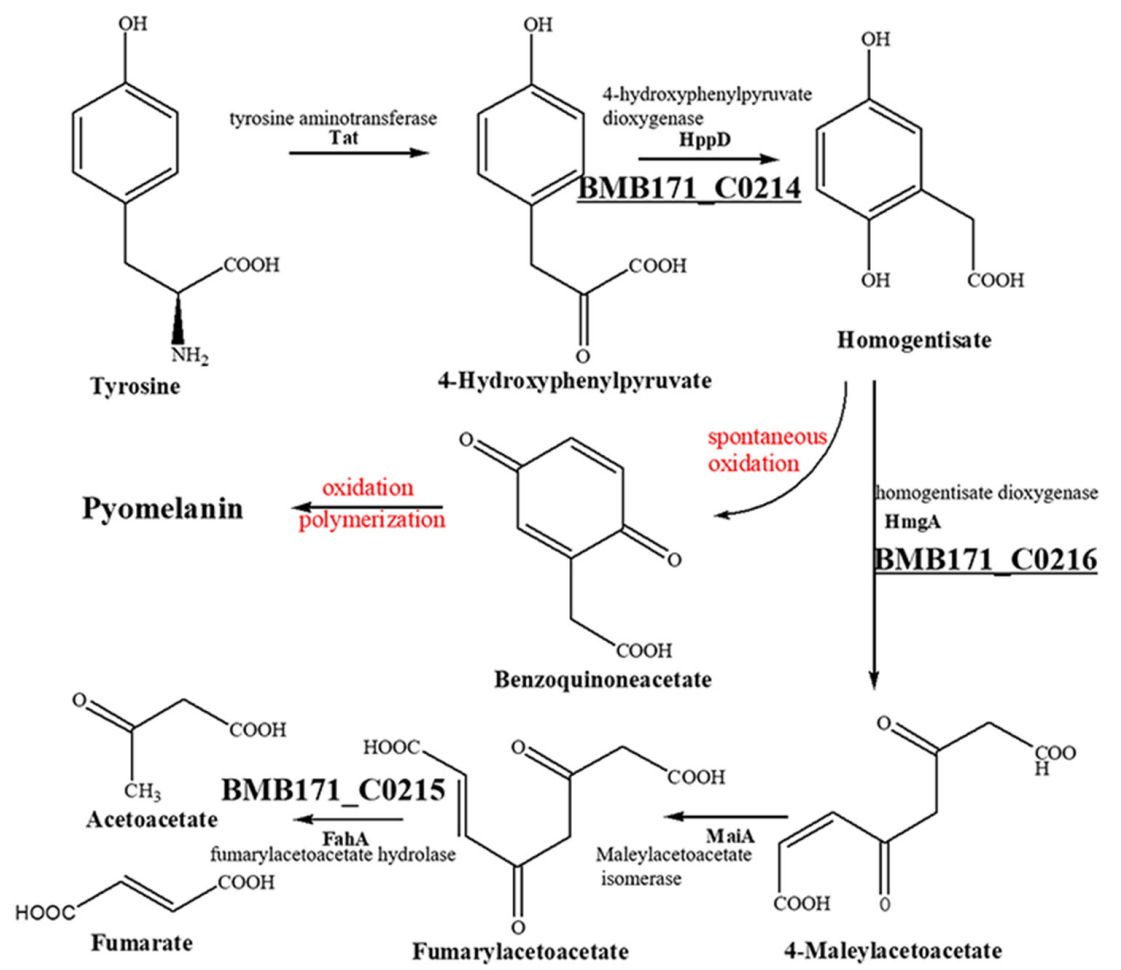

B

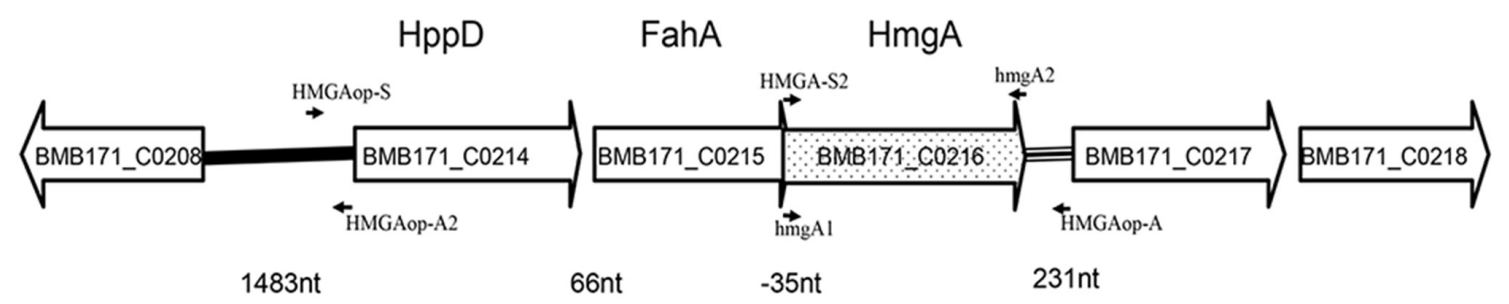

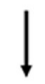

PCR with primers hmgAl and hmgA2, obtained the $h m g A$ gene fragment (1,173bp)

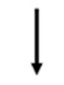

PCR with primers HMGA-

$\mathrm{S}$ and HMGAop-A,

obtained the complete

$h m g A$ operon, about $3.9 \mathrm{~kb}$
PCR with primers HMGAop-S and HMGAop-A2, obtained the promoter region (394bp)
PCR with primers HMGA-S2 and HMGAop-A, obtained the $h m g A$ gene and terminator region ( $1,405 \mathrm{bp})$
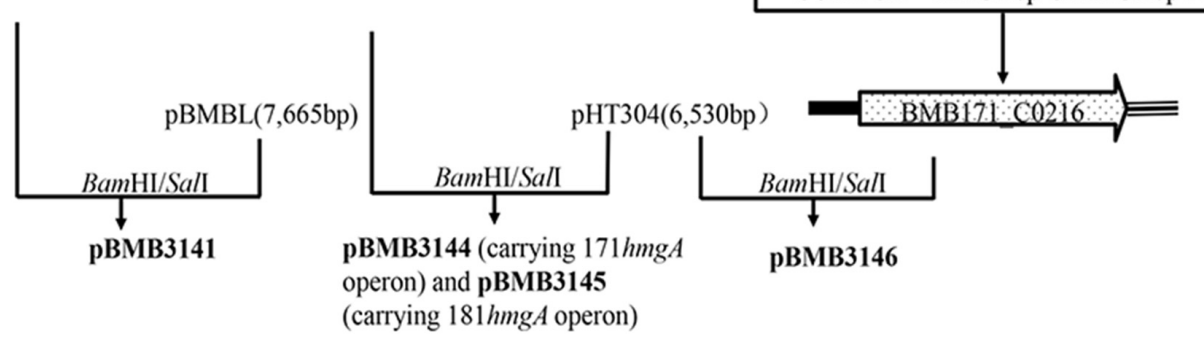

FIGURE 3 | Pathway for the catabolism of homogentisate. (A) L-Tyrosine metabolism pathway via homogentisate [modified from reference (Schmaler-Ripcke et al., 2009)]. (B) Organization of the genes putatively involved in the catabolism of homogentisate in $B$. thuringiensis BMB171 and scheme for the construction of plasmid vectors. The black arrows indicate the primers used here. Enzymes encoded by the respective genetic loci in B. thuringiensis BMB171 are BMB171_C0208, HAD superfamily hydrolase; BMB171_C0214, HppD, 4-hydroxyphenylpyruvate dioxygenase; BMB171_C0215, FahA, fumarylacetoacetate hydrolase; BMB171_C0216, 


\section{FIGURE 3 | Continued}

HmgA, homogentisate 1,2-dioxygenase; BMB171_C0217, amino acid permease; BMB171_C0218, MFS transporter. The vector pBMB3141, a derivative of $\mathrm{pBMBL}$, contained the $\mathrm{hmgA}$ gene (1,173 bp) amplified from the genomic DNA of BMB171 by using primers hmgA1 and hmgA2. The vector pBMB3144 carried the hmgA gene operon from BMB171, and the vector pBMB3145 carried the $h m g A$ gene operon from BMB181. The $h m g A$ gene operon region (about 3.9 kb) was amplified by using primers HMGAop-S and HMGAop-A. The vector pBMB3146, a derivative of pHT304, contained the transcription promoter region and the terminator region of the $h m g A$ gene operon and the $h m g A$ gene from BMB171. The promoter region (394 bp) was amplified from the genomic DNA of BMB171 by using primers HMGAop-S and HMGAop-A2. The $h m g A$ gene (1,173 bp) and the terminator region (232 bp) of the $h m g A$ gene operon was amplified from the genomic DNA of BMB171 by using primers HMGA-S2 and HMGAop-A.

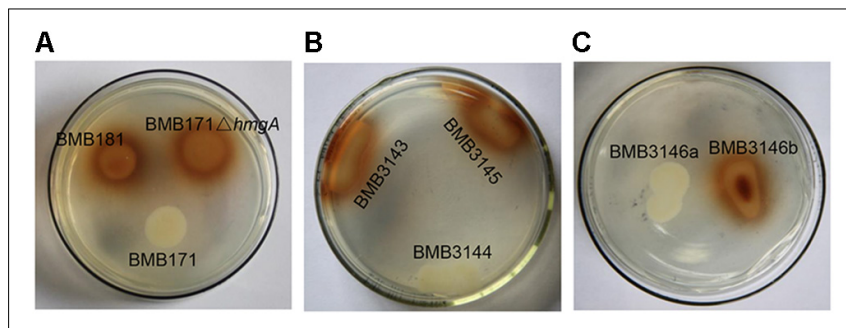

FIGURE 4 | Phenotypes of $B$. thuringiensis strains grown on LB agar plates. (A) Phenotypes of BMB171, BMB181, and BMB171 $4 h m g A$. (B) Phenotypes of BMB3143, BMB3144, and BMB3145. BMB3143, a derivative of $\mathrm{BMB} 171 \Delta h m g A$, contained pHT304, as a negative control; BMB3144, a derivative of BMB171 $A h m g A$, contained pBMB3144 harboring the $h m g A$ gene operon from BMB171; and BMB3145, a derivative of BMB171 $\Delta h m g A$, contained pBMB3145 harboring the $h m g A$ gene operon from BMB181. (C) Phenotypes of BMB3146a and BMB3146b. BMB3146a, a derivative of BMB181, contained pBMB3144; BMB3146b, a derivative of BMB181, contained pBMB3145.

by $\mathrm{HppD}$ and the oxidation of HGA to maleylacetoacetate (MAA) by HmgA. MAA is isomerized by maleylacetoacetate isomerase (MaiA) to fumarylacetoacetate, which is subsequently hydrolyzed by FahA to fumarate and acetoacetate (SchmalerRipcke et al., 2009; Valeru et al., 2009). The genes encoding these enzymes (HppD, HmgA, FahA, and MaiA) are adjacent on the chromosome of several organisms such as Aspergillus fumigatus, Pseudomonas chlororaphis, and Vibrio cholerae (Kang et al., 2008; Schmaler-Ripcke et al., 2009; Valeru et al., 2009). In the KEGG pathway database, three genes (BMB171_C1377, BMB171_C1351, and BMB171_C2647) are predicted to encode the aminotransferases involved in the conversion of L-tyrosine to 4-hydroxyphenylpyruvate. The three enzymes (HppD, HmgA, and FahA) in B. thuringiensis BMB171 are probably transcribed as part of an operon as indicated by the genomic analysis (Figure 3B). Analyzing the genome sequences of $B$. thuringiensis strains of different $\mathrm{H}$ serotypes, we found that the gene operon for L-Tyrosine degradation via HGA is located on every genome (data not shown). However, the genes responsible for the isomerization of MAA cannot be directly identified in $B$. thuringiensis by analyzing the genomic data. This implies that the genes responsible for the MAA metabolism of $B$. thuringiensis might be different from that of the reported genes. Even so, we found that the inactivation of HmgA leads to pyomelanin hyperproduction in $B$. thuringiensis strains. We speculate that $B$. thuringiensis strains have the potential to produce pyomelanin via HGA through the tyrosine metabolism pathway by inactivating HmgA.
HmgA is involved in the catabolism of the phenylalanine and tyrosine pathway. The structure of the human HmgA shows that the enzyme forms a hexamer arrangement consisting of a dimer of trimers (Titus et al., 2000). HmgA has been predicted to belong to the cupin-like superfamily ${ }^{1}$, which refers to a $\beta$-barrel structural domain, on the basis of the primary sequence. Two conserved histidine-containing motifs provide the signature sequence for the cupin superfamily (Dunwell et al., 2001). Despite the considerable variability of the HmgA proteins from different organisms in their primary amino acid sequences (from 22 to $65 \%$ ), the residues involved in coordinating $\mathrm{Fe}^{2+}$ are highly conserved in the HmgA proteins from bacteria, fungi, plants, invertebrates, and humans (Figure 5). Three residues, including two residues His298 and Glu305 in motif 1, and one residue His334 in motif 2, are responsible for the metal ion binding of the $171 \mathrm{HmgA}$ protein (Figure 5). Additionally, the secondary structure of $171 \mathrm{HmgA}$ is composed of many $\beta$-strands (Figure 5). In this study, we found that a G272E mutation in HmgA resulted in pigment overproduction in the $B$. thuringiensis strain BMB181. Residues 272-274 of HmgA form a loop structure connecting two $\beta$-strands ( $\beta 19$ and $\beta 20)$. Gly272 is highly conserved in different HmgAs and the equivalent in the human HmgA is Gly309 (Figure 5). A G309V missense mutation has previously been found in AKU patients (Nemethova et al., 2016). These findings suggest that the residue Gly272 plays a very important role in the enzyme activity. To verify whether other residues in the loops or adjacent to the loops have the same effect as the residue Gly272, we designed 14 single-point mutants. Nine of the residues (Gly89, Gly119, Gly128, Phe136, Pro245, His261, Asn300, Gly323, and Pro336) are located in different loop regions, and the other five (Asn116, Asp120, Lys219, Gly241, and His334) are located at the start or end of different $\beta$-strands adjacent to the loop-forming residues. The result shows that only six mutants (G128A, F136A, G241A, H261A, H334A, and P336A) have an effect on melanin production (Table 3 ). The activity of the enzyme can be disrupted by mutations in many different ways. Some will directly or indirectly affect the active site, others will interfere with the folding of the subunit, and some will affect intersubunit interactions (Rodriguez et al., 2000). The relationship between these residues and the catalytic activity needs to be further confirmed by biochemical characterization and crystal structure.

Previous studies have shown that residues in many different sites of homogentisate 1,2 dioxygenase from humans are essential for enzyme function (Rodriguez et al., 2000; Vilboux et al., 2009; Zatkova, 2011). For instance, single amino acid

\footnotetext{
${ }^{1}$ https://www.ncbi.nlm.nih.gov/cdd/
} 


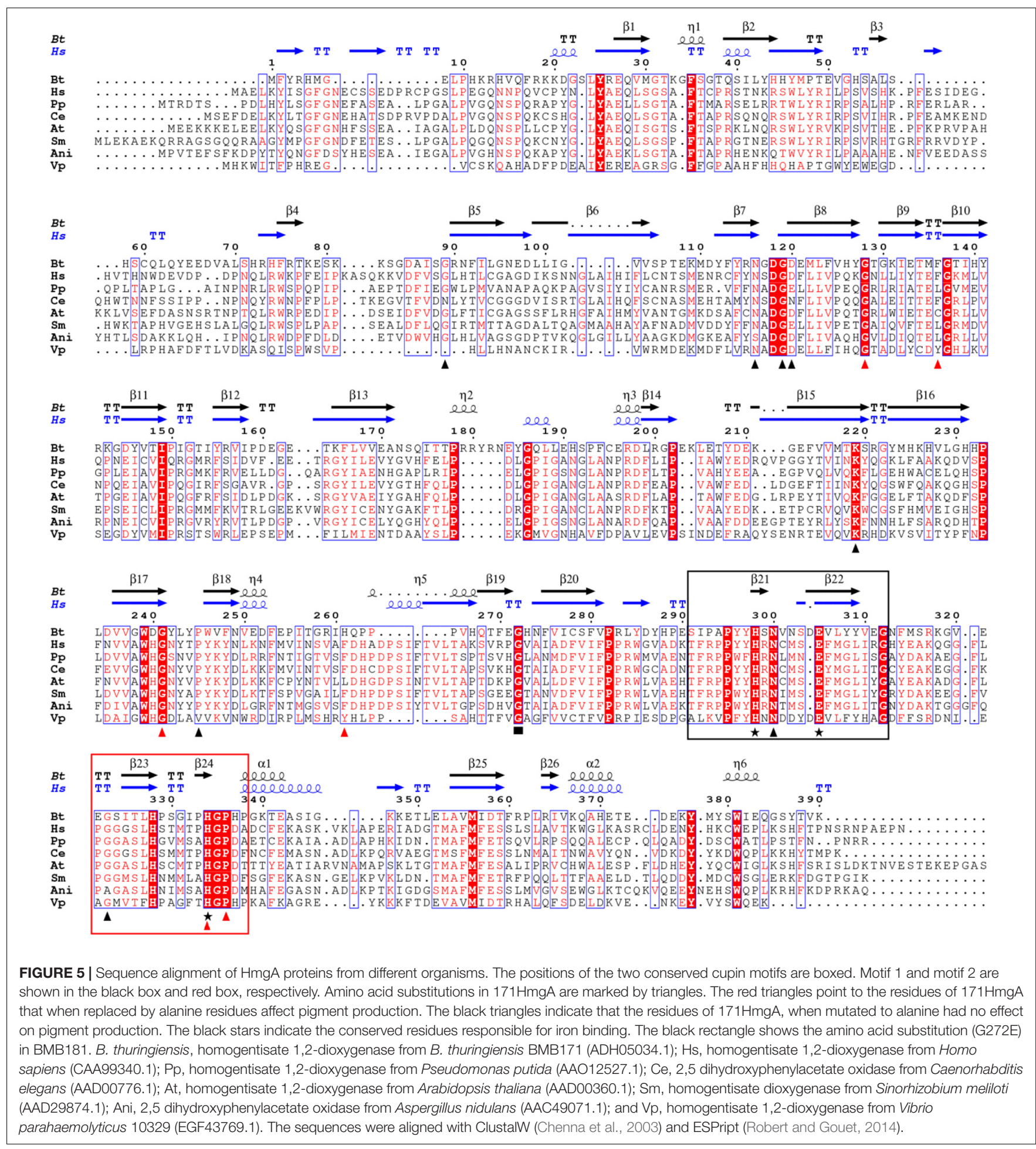

substitutions at different positions of $\mathrm{HmgA}$ can result in pyomelanin overproduction in AKU patients (Rodriguez et al., 2000; Vilboux et al., 2009; Zatkova, 2011). The deduced amino acid sequence of $171 \mathrm{HmgA}$ consists of 390 amino acids and shares $24 \%$ identity with the HmgA from humans. The singleresidue substitution in the 13 equivalents to the aforementioned
14 mutational residues in the human HmgA sequence would result in the loss of enzyme activity and pigment production (Table 3; Zatkova, 2011; Usher et al., 2015). These results indicate that five residues (G128, F136, G241, H334, and P336) are more conservative than the others (G89, N116, G119, D120, K219, P245, N300, and G323) in HmgA, and 
TABLE 3 | Site-directed mutagenesis in HmgA.

\begin{tabular}{|c|c|c|c|c|c|}
\hline $\begin{array}{l}\text { Transformants of } \\
\text { BMB171 ( } \Delta h m g A)\end{array}$ & Mutation position & $\begin{array}{l}\text { Amino acid } \\
\text { change }\end{array}$ & Phenotype $^{a}$ & $\begin{array}{l}\text { Equivalent residue(s) } \\
\text { in human HmgA }\end{array}$ & $\begin{array}{c}\text { Amino acid changes in AKU } \\
\text { patients (Zatkova, 2011; } \\
\text { Usher et al., 2015) }\end{array}$ \\
\hline BMB3147 & hmgA89 & G89A & $\mathrm{N}$ & $115 G$ & G115R \\
\hline BMB3148 & hmgA116 & N116A & $\mathrm{N}$ & $149 N$ & N149K \\
\hline BMB3149 & hmgA119 & G119A & $N$ & $152 \mathrm{G}$ & G152A \\
\hline BMB3150 & hmgA120 & D120A & $\mathrm{N}$ & $153 D$ & D153G \\
\hline BMB3151 & hmgA128 & G128A & $P$ & $161 \mathrm{G}$ & G161R \\
\hline BMB3152 & hmgA136 & F136A & $\mathrm{P}$ & $169 F$ & F169L \\
\hline BMB3153 & hmgA219 & K219A & $N$ & $248 K$ & K248E \\
\hline BMB3154 & hmgA241 & G241A & $\mathrm{P}$ & $270 G$ & G270R \\
\hline BMB3155 & hmgA245 & P245A & $\mathrm{N}$ & $274 \mathrm{P}$ & P274L \\
\hline BMB3156 & hmgA261 & H261A & $\mathrm{P}$ & $290 F$ & $?$ \\
\hline BMB3157 & hmgA300 & N300A & $N$ & $337 N$ & N337D \\
\hline BMB3158 & hmgA323 & G323A & $\mathrm{N}$ & $360 G$ & G360R/A \\
\hline BMB3159 & hmgA334 & H334A & $P$ & $371 \mathrm{H}$ & H371R \\
\hline BMB3160 & hmgA336 & P336A & $P$ & $373 P$ & P373L \\
\hline
\end{tabular}

${ }^{a} \mathrm{~N}$ : no pigment; $P$ : pigment.

b?: no single amino acid substitution was found in the residue F290 in AKU patients in the present study.

mutations in residues G128, F136, G241, H334, and P336 could affect the production of pyomelanin in both humans and B. thuringiensis.

Pyomelanin production has been studied in different bacteria. The accumulation of pyomelanin does not affect the growth characteristics nor the expression of key virulence factors of B. anthracis (Han et al., 2015). Notably, the mutant $P$. aeruginosa strain PA14 $\Delta \mathrm{hmgA}$ is significantly more virulent than the wild-type PA14 as PA14 $\Delta h m g A$ can kill the nematodes at a greatly accelerated rate compared with the wild-type PA14 (Harmer et al., 2015). Additionally, pyomelanin has been identified as the primary melanin produced by the A. media strain WS through the autoxidation and self-polymerization of HGA (Wang et al., 2015). The melanin produced by the strain WS serves as an excellent photoprotective agent for BTI against UV and sunlight radiation (Wan et al., 2007). A previous study has shown that the pigment produced by the $B$. thuringiensis strain BMB181 protects against UV radiation (Liu et al., 2013). To test the possible role of pyomelanin in $\mathrm{H}_{2} \mathrm{O}_{2}$ resistance, we estimated the viability of the culture of the strain BMB171 under $\mathrm{H}_{2} \mathrm{O}_{2}$ treatment and found that the supernatant from the mutant culture was able to protect the vegetative cells from the effect of $\mathrm{H}_{2} \mathrm{O}_{2}$ based on the growth curves (data not shown). We speculate that the pyomelanin produced by $B$. thuringiensis strains may have a significant synergistic effect on the crystal proteins against nematodes and other insect pests by not affecting the growth and the expression of key virulence factors of $B$. thuringiensis and protecting B. thuringiensis cells from the stresses such as $\mathrm{H}_{2} \mathrm{O}_{2}$ and $\mathrm{UV}$ during the life cycle.

In this study, we constructed several plasmid vectors for complementation analysis (Figure 3B). The plasmid pBMB3141 was constructed using the vector $\mathrm{pBMBL}$ (unpublished data) and the single $h m g A$ gene fragment for the complementation analysis. However, we found that the strain BMB181 could not completely restore the non-pigment phenotype with the plasmid pBMB3141 transformed into it, and the pigment appeared after culturing the transformant BMB3141 (a derivative of BMB181 containing pBMB3141) in LB medium for over $40 \mathrm{~h}$ (Figure 1B), suggesting that the promoter of the vector pBMBL, a sporulation-dependent promoter (BtI-BtII), is not suitable for this restoration test. To solve this problem, we tried to construct a plasmid vector that contained its own promoter and terminator region of the $h m g A$ gene for complementation analysis. As shown in Figure $\mathbf{3 B}$, the genes $h p p D$, fahA, and $h m g A$ are organized as an operon as indicated by the genomic analysis, and the transcription of the operon would be influenced with the $h m g A$ gene being interrupted by the kanamycin resistance cassette in the strain BMB171 $\Delta h m g A$. Thus, we constructed the plasmid vectors (Figure $3 \mathbf{B}$ ) that contained the complete $h m g A$ gene operon from strains BMB171 and BMB181 separately for the complementation analysis (Figures $4 \mathrm{~B}, \mathrm{C}$ ). The functions of HmgA variants in melanin production were also tested by the complementation analysis. We firstly constructed the vector pBMB3146 that contained the promoter and terminator regions of the $h m g A$ gene operon and the $h m g A$ gene (Figure 3B). All of the point mutations in HmgA were carried out by gene splicing by overlap extension PCR (SOE PCR) with pBMB3146 as the template. By introducing the plasmids containing different $h m g A$ gene variants into the pigmented strain BMB171 $\Delta h m g A$, the roles of different HmgA variants was preliminarily determined by the phenotypes (pigmented or non-pigmented) (Table 3). This indicates that some of these residues play important roles in enzyme activity. Nevertheless, the exact roles of these residues for the enzyme HmgA could not be 
determined based only on the aforementioned data. The difference between HmgA variants should be distinguished by catalytic mechanisms in further related research.

In summary, $B$. thuringiensis strains could produce pyomelanin via HGA upon the deactivation of the HmgA or the disruption of the $h m g A$ gene. We found that a G272E amino acid substitution in HmgA resulted in pigmentation. Several other residues in the loops or adjacent to the loops have the same effect as the residue Gly272 on the enzyme activity and melanin production. It is possible to generate more mutations in the $h m g A$ gene and reintroduce them into $B$. thuringiensis and in many cases obtain a mutant phenotype (pigment overproducer). This approach has potential use to producing $B$. thuringiensis strains more resistant to UV. This is also an interesting model to study the human gene responsible for alkaptonuria.

\section{REFERENCES}

Ahmad, S., Lee, S. Y., Khan, R., Kong, H. G., Son, G. J., Roy, N., et al. (2017). Identification of a gene involved in the negative regulation of pyomelanin production in Ralstonia solanacearum. J. Microbiol. Biotechnol. 27, 1692-1700. doi: 10.4014/jmb.1705.05049

Andrup, L., Damgaard, J., and Wassermann, K. (1993). Mobilization of small plasmids in Bacillus thuringiensis subsp. israelensis is accompanied by specific aggregation. J. Bacteriol. 175, 6530-6536. doi: 10.1128/jb.175.20.6530-6536. 1993

Arantes, O., and Lereclus, D. (1991). Construction of cloning vectors for Bacillus thuringiensis. Gene 108, 115-119. doi: 10.1016/0378-1119(91)90495-W

Arias-Barrau, E., Olivera, E. R., Luengo, J. M., Fernandez, C., Galan, B., Garcia, J. L., et al. (2004). The homogentisate pathway: a central catabolic pathway involved in the degradation of L-phenylalanine, L-tyrosine, and 3-hydroxyphenylacetate in Pseudomonas putida. J. Bacteriol. 186, 5062-5077. doi: 10.1128/JB.186.15. 5062-5077.2004

Chenna, R., Sugawara, H., Koike, T., Lopez, R., Gibson, T. J., Higgins, D. G., et al. (2003). Multiple sequence alignment with the Clustal series of programs. Nucleic Acids Res. 31, 3497-3500. doi: 10.1093/nar/gkg500

Dunwell, J. M., Culham, A., Carter, C. E., Sosa-Aguirre, C. R., and Goodenough, P. W. (2001). Evolution of functional diversity in the cupin superfamily. Trends Biochem. Sci. 26, 740-746. doi: 10.1016/S0968-0004(01)01981-8

Fernandez-Canon, J. M., and Penalva, M. A. (1995). Molecular characterization of a gene encoding a homogentisate dioxygenase from Aspergillus nidulans and identification of its human and plant homologues. J. Biol. Chem. 270, 21199-21205. doi: 10.1074/jbc.270.36.21199

Gonyar, L. A., Fankhauser, S. C., and Goldberg, J. B. (2015). Single amino acid substitution in homogentisate 1,2-dioxygenase is responsible for pigmentation in a subset of Burkolderia cepacia complex isolates. Environ. Microbiol. Rep. 7, 180-187. doi: 10.1111/1758-2229.12217

Green, M. R., and Sambrook, J. (2012). Molecular Cloning: A Laboratory Manual. New York, NY: Cold Spring Harbor Laboratory Press.

Guerout-Fleury, A. M., Shazand, K., Frandsen, N., and Stragier, P. (1995). Antibiotic-resistance cassettes for Bacillus subtilis. Gene 167, 335-336. doi: 10. 1016/0378-1119(95)00652-4

Han, H., Iakovenko, L., and Wilson, A. C. (2015). Loss of homogentisate 1,2dioxygenase activity in Bacillus anthracis results in accumulation of protective pigment. PLoS One 10:e0128967. doi: 10.1371/journal.pone.0128967

Harmer, C. J., Wynn, M., Pinto, R., Cordwell, S., Rose, B. R., Harbour, C., et al. (2015). Homogentisate 1-2-dioxygenase downregulation in the chronic persistence of Pseudomonas aeruginosa Australian epidemic strain-1 in the CF lung. PLoS One 10:e0134229. doi: 10.1371/journal.pone.0134229

He, J., Shao, X., Zheng, H., Li, M., Wang, J., Zhang, Q., et al. (2010). Complete genome sequence of Bacillus thuringiensis mutant strain BMB171. J. Bacteriol. 192, 4074-4075. doi: 10.1128/JB.00562-10

Heinekamp, T., Thywissen, A., Macheleidt, J., Keller, S., Valiante, V., and Brakhage, A. A. (2012). Aspergillus fumigatus melanins: interference with the

\section{AUTHOR CONTRIBUTIONS}

WY, LR, and MS designed the research. WY and JT performed the research. WY, JZ, LR, DP, and MS analyzed the data. WY, LR, and MS wrote the manuscript.

\section{FUNDING}

This work was supported by grants from the National Key R\&D Program of China (2017YFD0201201), National Natural Science Foundation of China (31670085 and 31600005), China 948 Program of Ministry of Agriculture (2016-X21), and Fundamental Research Funds for the Central Universities (2662017PY094).

host endocytosis pathway and impact on virulence. Front. Microbiol. 3:440. doi: $10.3389 /$ fmicb. 2012.00440

Higuchi, R., Krummel, B., and Saiki, R. K. (1988). A general method of in vitro preparation and specific mutagenesis of DNA fragments: study of protein and DNA interactions. Nucleic Acids Res. 16, 7351-7367. doi: 10.1093/nar/16. 15.7351

Hoti, S. L., and Balaraman, K. (1993). Formation of melanin pigment by a mutant of Bacillus thuringiensis H-14. J. Gen. Microbiol. 139, 2365-2369. doi: 10.1099/ 00221287-139-10-2365

Jallouli, W., Sellami, S., Sellami, M., and Tounsi, S. (2014). Efficacy of olive mill wastewater for protecting Bacillus thuringiensis formulation from UV radiations. Acta Trop. 140C, 19-25. doi: 10.1016/j.actatropica.2014.07.016

Kang, B. R., Han, S. H., Cho, S. M., Anderson, A. J., Kim, I. S., Park, S. K., et al. (2008). Characterization of a homogentisate dioxygenase mutant in Pseudomonas chlororaphis O6. Curr. Microbiol. 56, 145-149. doi: 10.1007/ s00284-007-9075-7

Liu, F. X., Yang, W. J., Ruan, L. F., and Sun, M. (2013). A Bacillus thuringiensis host strain with high melanin production for preparation of light-stable biopesticides. Ann. Microbiol. 63, 1131-1135. doi: 10.1007/s13213-012-0570-0

Liu, X. Y., Ruan, L. F., Hu, Z. F., Peng, D. H., Cao, S. Y., Yu, Z. N., et al. (2010). Genome-wide screening reveals the genetic determinants of an antibiotic insecticide in Bacillus thuringiensis. J. Biol. Chem. 285, 39191-39200. doi: 10. 1074/jbc.M110.148387

Liu, Y. T., Sui, M. J., Ji, D. D., Wu, I. H., Chou, C. C., and Chen, C. C. (1993). Protection from ultraviolet irradiation by melanin of mosquitocidal activity of Bacillus thuringiensis var. israelensis. J. Invertebr. Pathol. 62, 131-136. doi: 10.1006/jipa.1993.1088

Manasherob, R., Ben-Dov, E., Xiaoqiang, W., Boussiba, S., and Zaritsky, A. (2002). Protection from UV-B damage of mosquito larvicidal toxins from Bacillus thuringiensis subsp. israelensis expressed in Anabaena PCC 7120. Curr. Microbiol. 45, 217-220. doi: 10.1007/s00284-001-0106-5

Millucci, L., Spreafico, A., Tinti, L., Braconi, D., Ghezzi, L., Paccagnini, E., et al. (2012). Alkaptonuria is a novel human secondary amyloidogenic disease. Biochim. Biophys. Acta 1822, 1682-1691. doi: 10.1016/j.bbadis.2012.07.011

Nemethova, M., Radvanszky, J., Kadasi, L., Ascher, D. B., Pires, D. E., Blundell, T. L., et al. (2016). Twelve novel HGD gene variants identified in 99 alkaptonuria patients: focus on 'black bone disease' in Italy. Eur. J. Hum. Genet. 24, 66-72. doi: 10.1038/ejhg.2015.60

Nosanchuk, J. D., and Casadevall, A. (2003). The contribution of melanin to microbial pathogenesis. Cell Microbiol 5, 203-223. doi: 10.1046/j.1462-5814. 2003.00268.x

Nosanchuk, J. D., and Casadevall, A. (2006). Impact of melanin on microbial virulence and clinical resistance to antimicrobial compounds. Antimicrob. Agents Chemother. 50, 3519-3528. doi: 10.1128/AAC.00545-06

Peng, D., Luo, Y., Guo, S., Zeng, H., Ju, S., Yu, Z., et al. (2009). Elaboration of an electroporation protocol for large plasmids and wild-type strains of Bacillus thuringiensis. J. Appl. Microbiol. 106, 1849-1858. doi: 10.1111/j.1365-2672.2009. 04151.x 
Plonka, P. M., and Grabacka, M. (2006). Melanin synthesis in microorganismsbiotechnological and medical aspects. Acta Biochim. Pol. 53, 429-443.

Pusztai, M., Fast, P., Gringorten, L., Kaplan, H., Lessard, T., and Carey, P. R. (1991). The mechanism of sunlight-mediated inactivation of Bacillus thuringiensis crystals. Biochem. J. 273(Pt 1), 43-47. doi: 10.1042/bj2730043

Robert, X., and Gouet, P. (2014). Deciphering key features in protein structures with the new ENDscript server. Nucleic Acids Res. 42, W320-W324. doi: 10. 1093/nar/gku316

Rodriguez, J. M., Timm, D. E., Titus, G. P., Beltran-Valero, De Bernabe, D., Criado, O., et al. (2000). Structural and functional analysis of mutations in alkaptonuria. Hum. Mol. Genet. 9, 2341-2350. doi: 10.1093/oxfordjournals. hmg.a018927

Rodriguez-Rojas, A., Mena, A., Martin, S., Borrell, N., Oliver, A., and Blazquez, J. (2009). Inactivation of the hmgA gene of Pseudomonas aeruginosa leads to pyomelanin hyperproduction, stress resistance and increased persistence in chronic lung infection. Microbiology 155(Pt 4), 1050-1057. doi: 10.1099/mic. 0.024745-0

Ruan, L., Huang, Y., Zhang, G., Yu, D., and Ping, S. (2002). Expression of the mel gene from Pseudomonas maltophilia in Bacillus thuringiensis. Lett. Appl. Microbiol. 34, 244-248. doi: 10.1046/j.1472-765x.2002.01049.x

Ruan, L., Yu, Z., Fang, B., He, W., Wang, Y., and Shen, P. (2004). Melanin pigment formation and increased UV resistance in Bacillus thuringiensis following high temperature induction. Syst. Appl. Microbiol. 27, 286-289. doi: 10.1078/07232020-00265

Sanchis, V., Gohar, M., Chaufaux, J., Arantes, O., Meier, A., Agaisse, H., et al. (1999). Development and field performance of a broad-spectrum nonviable asporogenic recombinant strain of Bacillus thuringiensis with greater potency and UV resistance. Appl. Environ. Microbiol. 65, 4032-4039.

Sansinenea, E., and Ortiz, A. (2015). Melanin: a photoprotection for Bacillus thuringiensis based biopesticides. Biotechnol. Lett. 37, 483-490. doi: 10.1007/ s10529-014-1726-8

Saxena, D., Ben-Dov, E., Manasherob, R., Barak, Z., Boussiba, S., and Zaritsky, A. (2002). A UV tolerant mutant of Bacillus thuringiensis subsp. kurstaki producing melanin. Curr. Microbiol. 44, 25-30. doi: 10.1007/s00284-0010069-6

Schmaler-Ripcke, J., Sugareva, V., Gebhardt, P., Winkler, R., Kniemeyer, O., Heinekamp, T., et al. (2009). Production of pyomelanin, a second type of melanin, via the tyrosine degradation pathway in Aspergillus fumigatus. Appl. Environ. Microbiol. 75, 493-503. doi: 10.1128/AEM.02077-08

Sudakin, D. L. (2003). Biopesticides. Toxicol. Rev. 22, 83-90. doi: 10.2165/ 00139709-200322020-00003

Titus, G. P., Mueller, H. A., Burgner, J., Rodriguez, De Cordoba, S., Penalva, M. A., et al. (2000). Crystal structure of human homogentisate dioxygenase. Nat. Struct. Biol. 7, 542-546. doi: 10.1038/76756
Turick, C. E., Knox, A. S., Becnel, J. M., Ekechukwu, A. A., and Milliken, C. E. (2010). "Properties and function of pyomelanin," in Biopolymers, ed. M. Elnashar (Rijeka: InTech), 449-472.

Usher, J. L., Ascher, D. B., Pires, D. E., Milan, A. M., Blundell, T. L., and Ranganath, L. R. (2015). Analysis of HGD gene mutations in patients with alkaptonuria from the United Kingdom: identification of novel mutations. JIMD Rep. 24, 3-11. doi: 10.1007/8904-2014-380

Valeru, S. P., Rompikuntal, P. K., Ishikawa, T., Vaitkevicius, K., Sjoling, A., Dolganov, N., et al. (2009). Role of melanin pigment in expression of Vibrio cholerae virulence factors. Infect. Immun. 77, 935-942. doi: 10.1128/IAI.00 929-08

Vilboux, T., Kayser, M., Introne, W., Suwannarat, P., Bernardini, I., Fischer, R., et al. (2009). Mutation spectrum of homogentisic acid oxidase (HGD) in alkaptonuria. Hum. Mutat. 30, 1611-1619. doi: 10.1002/humu.21120

Wan, X., Liu, H. M., Liao, Y., Su, Y., Geng, J., Yang, M. Y., et al. (2007). Isolation of a novel strain of Aeromonas media producing high levels of DOPA-melanin and assessment of the photoprotective role of the melanin in bioinsecticide applications. J. Appl. Microbiol. 103, 2533-2541. doi: 10.1111/j.1365-2672.2007. 03502.x

Wang, H., Qiao, Y., Chai, B., Qiu, C., and Chen, X. (2015). Identification and molecular characterization of the homogentisate pathway responsible for pyomelanin production, the major melanin constituents in Aeromonas media WS. PLoS One 10:e0120923. doi: 10.1371/journal.pone.0120923

Zatkova, A. (2011). An update on molecular genetics of Alkaptonuria (AKU). J. Inherit. Metab. Dis. 34, 1127-1136. doi: 10.1007/s10545-0119363-Z

Zeng, Z., Cai, X., Wang, P., Guo, Y., Liu, X., Li, B., et al. (2017). Biofilm formation and heat stress induce pyomelanin production in deep-sea Pseudoalteromonas sp. SM9913. Front. Microbiol. 8:1822. doi: 10.3389/fmicb.2017.01822

Zhang, J. T., Yan, J. P., Zheng, D. S., Sun, Y. J., and Yuan, Z. M. (2008). Expression of mel gene improves the UV resistance of Bacillus thuringiensis. J. Appl. Microbiol. 105, 151-157. doi: 10.1111/j.1365-2672.2008.03729.x

Conflict of Interest Statement: The authors declare that the research was conducted in the absence of any commercial or financial relationships that could be construed as a potential conflict of interest.

Copyright (C) 2018 Yang, Ruan, Tao, Peng, Zheng and Sun. This is an open-access article distributed under the terms of the Creative Commons Attribution License (CC BY). The use, distribution or reproduction in other forums is permitted, provided the original author(s) and the copyright owner(s) are credited and that the original publication in this journal is cited, in accordance with accepted academic practice. No use, distribution or reproduction is permitted which does not comply with these terms. 